\title{
How to Improve the Quality of Raw Milk in China
}

\author{
Li Zhang ${ }^{1, a^{*}}$, Yunxian Hou, b ${ }^{1, b}$ Chang Liu ${ }^{1, c}$ and Wenzhuo Liang ${ }^{1, d}$ \\ ${ }^{1}$ College of Economics \& Management, China Agricultural University, Beijing 100083, China \\ azhangli323alive@163.com, 'bhouyunxianz2163, com, 'larry33bird@126.com, \\ diangwenzhuo917@163.com
}

\section{Keywords: Raw Milk; Techniques Support; Quality Improvement; Evolutionarily Stable Strategy}

\begin{abstract}
At present, raw milk is mainly supplied by smallholder dairy farmers or small-scale feeding bases in China. Considering liquid characteristics of raw milk and its current acquisition mode, the paper analyzes the feasibility of quality improvement cooperation between the dairy farmers and processing companies by the means of Multi-groups replicator dynamics model, supposing the processing companies provide techniques support to dairy farmers for the quality improvement of raw milk. This paper explores the strategy choices of dairy farmers and processing companies, and also investigates the main factors of cooperation between the two groups. Results show that the two groups' strategic choices are not only affected by the incomes change caused by quality improvement of raw milk, but also influenced by the R\&D costs or execution costs of the techniques. Finally, we present some suggestion to promote the cooperation between the two groups.
\end{abstract}

\section{Introduction}

Quality is one of the key competitive dimensions of a brand, and is an important feature that impacts current and future profits. As more and more companies source from different suppliers, the suppliers' component and all parties involved contribute to the overall quality of the goods. So quality management has been one of the most important aspects in operations management [1]. Just like other products, high quality dairy products require high quality raw milk. Whether the defects are due to the process or raw milk, the break-down of dairy product will not only reduce profits both of the processing companies and dairy farmers as well as affecting the industry development, but also do harm to consumer's health. As the main reason of the incident of Sanlu in 2008 is the defects of raw milk, it is important that raw milk be produced and handled in quality.

At present, raw milk is mainly supplied by smallholder dairy farmers or small-scale feeding bases in China. Unqualified farmers, lacking of quality-control awareness and weakness of feeding technique are the potential causes of quality defects in raw milk and dairy products. Even though the government and some powerful processing companies offer techniques of breeding and diseases precaution to dairy farmers for free for the improvement of raw milk, however, those techniques are not promoted and applied eventually because of execution costs and interest temptation. Besides, those common problems in most milk stations in China, such as lower automatic control level, outdated techniques and equipment, etc. lead to the fact that quality tests are only normal tests. Furthermore, neither the milk station's routine tests nor the processing companies' professional tests can identify all the potential problems of raw milk for the probable error of techniques and equipment, which induces the dairy farmers' speculative behavior directly, and the costs of quality improvement and the interest temptation aggravate this behavior.

\section{Literature Review}

Game theory was first used in studies of food safety by Mand [2] to study the relationship between the quality safe and governance structures of the agricultural products, and he put forward the opinions that agricultural products' quality could be improved with appropriate game theory. Now, for the academic study on the quality-improvement in food supply chain, both the non-cooperation game 
theory and traditional cooperation game theory are employed, such as oligopoly game model , repeated game model, dynamic game model with incomplete information and supervise game model [3-8]. However, most of these works are limited to recommending how the firms control their suppliers' quality through incoming inspection and cost penalties. These approaches, although effective, leave quality improvement to the suppliers. Furthermore, the basic assumptions of these models and researches were perfect rationality of game participants, that is to say, the participants had the ability of 'Infinite regression inference' and 'Never making mistakes'.

In practice, it is difficult to achieve the assumptions of perfect rationality. The evolutionary game theory not only combines the game theory and the dynamic evolution process organically, but also divests the basic assumption of perfect rationality of the game players. The continuous time replicator dynamics model, depicting the variation tendency of the group behavior with limited rationality well [9], was wildly used in the quality-improvement of agricultural products and food [2,10,11]. To ensure high product quality, more and more companies take a proactive role in quality improvement and invest directly in their suppliers' quality improvement, such as Techtronic Industries (TTI), Homelite, Milwaukee, and Ryobi. To ensure high product quality, TTI often forms and sends quality teams to help its suppliers improve their quality [1].

Given these facts, this paper aims to analyze the feasibility quality- improvement cooperation between the dairy farmers and the processing companies, e.g. is there quality-improvement cooperation between processing companies and dairy farmers? And if so, how to promote and achieve the cooperation? To answer these questions. We analyze the variation of strategic choices of the dairy farmers and the processing companies with the Multi-groups replicator dynamics model, to discuss the quality cooperation between the two groups.

\section{Model Formulation}

Assumption and Notation. To get high quality raw milk from dairy farmers, the processing companies may take their advantage of the techniques, capital and information to invest in the research of quality-improvement techniques of raw milk quality and popularize in the dairy farmers or not, so the strategies set of processing companies is ('R\&D', 'Not R\&D'), the dairy farmers may accept the techniques support or not, and the strategies set of dairy farmers is ('Accept', 'Not accept').

We denote the quality level of raw milk and per unit production cost by $\beta$ and ${ }^{c_{f}}$ respectively with the traditional breeding and epidemic prevention techniques at present, and $\beta \in[0,1)$, assuming $\alpha$ the probability that a unqualified part is correctly identified by incoming inspection, $\alpha \in[0,1)$.The unit price of buying raw milk is $p_{d}$. The processing cost of the processing companies is $c_{m}$ and the dairy products will be sold at the price $p_{m}$. If the dairy products produced with unqualified raw milk, the processing companies will lose 1 . The average R\&D costs of techniques support per unit raw milk is ${ }^{c_{m}}$, and it will cost the farmers $c_{f}$ to execute the techniques, and the quality of the raw milk will rise to $\gamma$.

Let $y$ and $(1-y)$ denote the ratio of the processing companies who choose the strategy of 'R\&D' and 'Not R\&D', while the ratio of dairy farmers who choose the strategy of 'Accept' and 'Not accept' are $x$ and $(1-x)$ at present respectively. The asymmetric game pay-off matrix of the two groups is shown in Table 1 ,

Table 1 The pay-off matrix of the dairy farmers and processing companies

\begin{tabular}{l|c|c|c|}
\multicolumn{5}{c|}{ Dairy Farmers } \\
\cline { 2 - 4 } $\begin{array}{l}\text { Processing } \\
\text { companies }\end{array}$ & $\begin{array}{c}\text { R \& D } \\
\text { Not R \& D }\end{array}$ & $M, N$ & Accept \\
\cline { 2 - 4 } & & $P+c_{m}^{\prime}, Q-c_{f}^{\prime}$ & $P+c_{m}^{\prime}, Q$ \\
\hline
\end{tabular}


Where $\quad M=[1-\alpha(1-\gamma)]\left(P_{m}-c_{m}\right)-(1-\alpha)(1-\gamma) l-c_{m}^{\prime}$

$$
N=[1-\alpha(1-\gamma)] P_{d}-c_{f}-c_{f},
$$
$P=[1-\alpha(1-\beta)]\left(P_{m}-c_{m}\right)-(1-\alpha)(1-\beta) l-c_{m} \quad$ and $\quad Q=[1-\alpha(1-\beta)] P_{d}-c_{f} \quad, \quad$ also, $M-P=(\gamma-\beta)\left[\alpha\left(P_{m}-c_{m}\right)-(1-\alpha) l\right]>0$ due to $\gamma>\beta$.

The expected revenues of the processing companies who choose the strategy of 'R\&D' or 'Not R\&D' is $E M I=x M+(1-x) P$ or $E M N=x\left(P+c_{m}^{\prime}\right)+(1-x)\left(P+c_{m}^{\prime}\right)=\left(P+c_{m}^{\prime}\right)$ respectively. So the average expected revenue of the processing companies group is $E M=y x M+y(1-x) P+(1-y)\left(P+c_{m}^{\prime}\right)$.

Similarly, the expected revenues of dairy farmers who choose the strategy of 'Accept' or 'Not accept' are $E F A^{M}=y N+(1-y)\left(Q-c_{f}^{\prime}\right)$ or $E F N^{M}=y Q+(1-y) Q=Q$ respectively. And the average expected revenue of the farmer group is given as $E F^{\prime \prime}=x y N+x(1-y)\left(Q-c_{f}^{\prime}\right)+(1-x) Q$.

Multi-groups Replicator Dynamics. Proposition 2: The evolution tendency of processing companies who choose the strategy of 'R\&D' in the Multi-groups replicator dynamics model has the following properties.

$$
x=\frac{c_{m}^{\prime}}{(\gamma-\beta)\left[\alpha\left(P_{m}-c_{m}\right)-(1-\alpha) l\right]}, y \equiv 0 \text {, the strategic choices of dairy farmers will have }
$$
no influence on the choices of the processing companies as in Fig. 1 (a).

(2) If $\frac{c_{m}^{\prime}}{(\gamma-\beta)\left[\alpha\left(P_{m}-c_{m}\right)-(1-\alpha) l\right]}>x, y=0$ is the evolutionary stable point, 'Not R\&D' will be the ESS of the processing companies as in Fig.1(c). If the incomes change caused by the dairy farmers who choose the strategy of 'Accept' is lower than the R\&D costs, the ratio will decrease until all of the processing companies choose the strategy of 'Not R\&D'.

(3) If $\frac{c_{m}}{(\gamma-\beta)\left[\alpha\left(P_{m}-c_{m}\right)-(1-\alpha) l\right]}<x<1, y=1$ is the evolutionary stable point, 'R \& D' will be the ESS of the processing companies as in Fig.1(b). If the incomes change caused by the dairy farmers who choose the strategy of 'Accept' is more than the R\&D costs of techniques support, the ratio will increase until all of the processing companies choose the strategy of ' $R \& D$ '.

The phase plot of the dynamic trend of processing companies group is depicted in Fig. 1

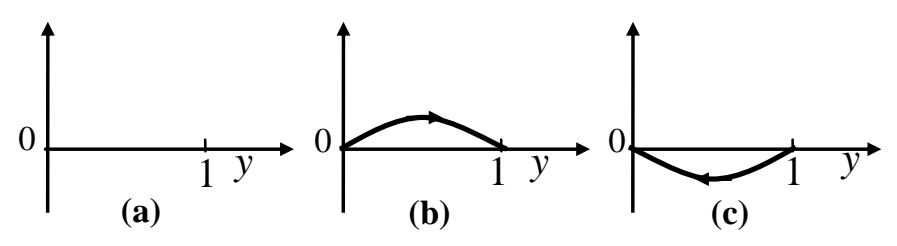

Figure 1. The phase plot of the dynamic trend of processing companies group

Proposition 3: The evolution tendency of the ratio of the dairy farmers who choose the strategy of 'Accept' within the model of Multi-groups replicator dynamics model has the following properties.

(1) If $y=\frac{c_{f}}{\alpha(\gamma-\beta) P_{d}}$, the strategic choices of the dairy farmers will not be influenced by the choices of processing companies, and stable with the variation of ${ }^{y}$ as in Fig. 2 (a).

(2) If $\frac{c_{f}}{\alpha(\gamma-\beta) P_{d}}>y, x=0$ is the evolutionary stable point, 'Not Accept' will be the ESS of the dairy farmers as in Fig.2(c). If the incomes change caused by the techniques support is less than the execution costs, the ratio will decrease until all of the dairy farmers choose the strategy of 'Not Accept'. 
(3) If $\frac{c_{f}}{\alpha(\gamma-\beta) P_{d}}<y<1, x=1$ is the evolutionary stable point, 'Accept' will be the ESS of the dairy farmers as in Fig. 2 (b). If the incomes change caused by the techniques support is more than the execution costs of techniques, the ratio will increase until all of the dairy farmers choose the strategy of 'Accept'.

The phase plot of the dynamic trend of dairy farmers group is depicted in Fig. 2

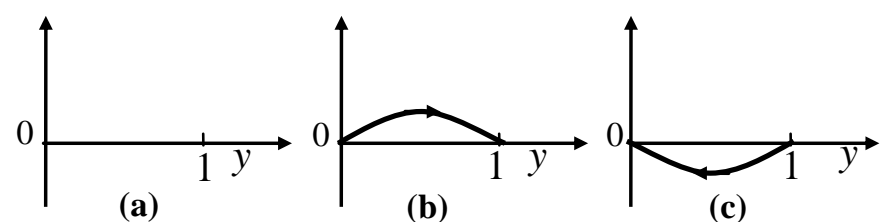

Figure 2. The phase plot of the dynamic trend of dairy farmers group

The phase plot of the dynamic trend is depicted in Fig. 3 after combining the replicator dynamics of the two groups.

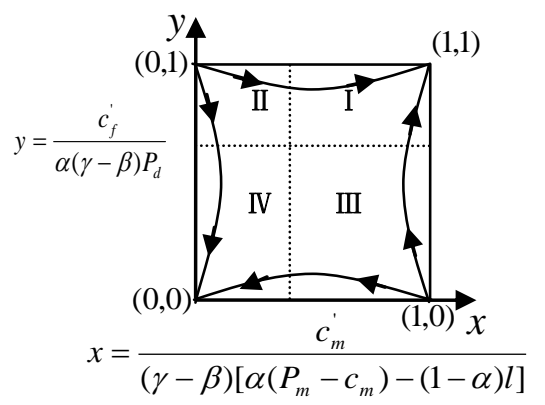

Figure 3. The phase plot of the dynamic trend of the two groups

The coordinate plane is divided into four parts by the two curves: $y=\frac{c_{f}^{\prime}}{\alpha(\gamma-\beta) P_{d}}$ and $x=\frac{c}{(\gamma-\beta)\left[\alpha\left(P_{m}-c_{m}\right)-(1-\alpha) l\right]}$.If and only if the ratios of the two groups fall in the region I simultaneously, both of dairy farmers and processing companies who choose the strategy of 'Accept' and 'R\&D' will raise constantly, the final state of evolution will be the ESS ('R\&D', 'Accept'), the quality of the raw milk will be improved with the strengthen of quality-improvement cooperation between the two groups. However, the improvement of the quality of raw milk will be held back because of the non-cooperation of processing companies, dairy farmers or both of them respectively, if the initial state falls in the region II, III, or IV.

\section{Simulation}

We assume $\beta=0.6, \gamma=0.7, \alpha=0.8, r=500, c_{m}=1000,1=1000, c_{f}^{\prime}=3$ and $c_{m}^{\prime}=1$. According to the facts of investigating and model analysis, we certificated the evolutionary trends of Group dynamic strategic choice between the processing companies and dairy farmers. Fig. 4(a), 4(b), 4(c) and 4(d) represent the ratios of the two groups falling in the region I , II, III and IV respectively. 

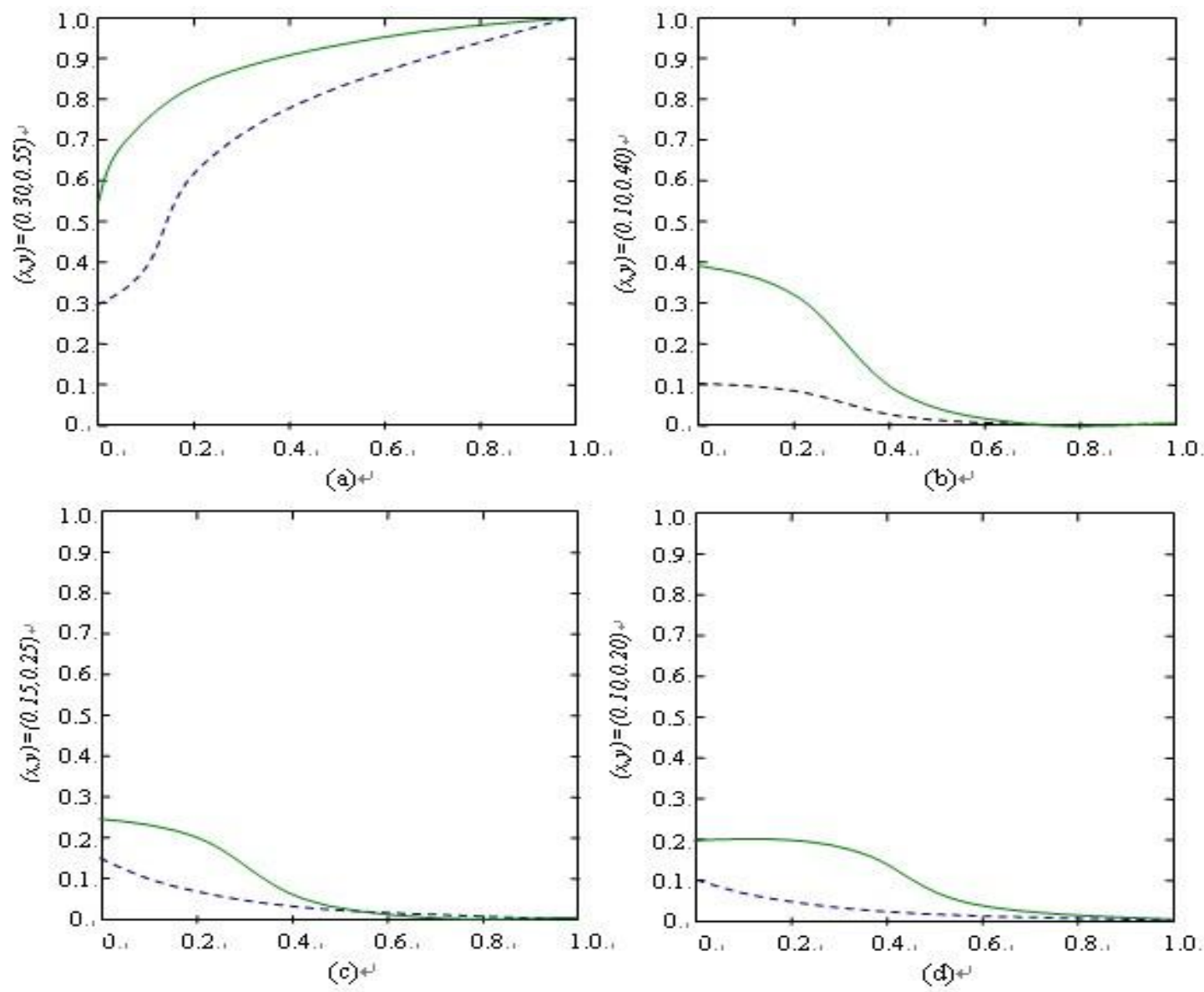

Figure 4. The evolutionary trends of Group dynamic strategic choice

\section{Conclusions}

In the Multi-groups Replicator Dynamics model of dairy farmers and processing companies, the strategic choices of the processing companies and dairy farmers are interacting with each other, and the ESS of the two groups are not only impacted by the R\&D costs and execution costs of the techniques, but also influenced by the ratios of dairy farmers and processing companies who choose to collaborate at present. Raising the incomes change caused by quality improvement of raw milk is the best way to motivate the dairy farmers and processing companies. From the processing companies' perspective, increasing consumers' claim and punishment would magnify the influence that raw milk quality have on processing companies' income. Apart from this, the subsidy for the $\mathrm{R} \& \mathrm{D}$ costs of techniques is another method to encourage processing companies to involve in the quality- improvement of the raw milk. Dairy farmers may be interested in the effectiveness and the execution costs of techniques which affect their incomes directly caused by quality improvement of raw milk. Compared with the direct cash subsidy traditionally, free techniques support would be more effective to promote popularization of the techniques among dairy farmers, that is to say, if the government want to invest in quality-improvement of dairy products, it would be better to subsidize the processing companies rather than the dairy farmers for techniques support.

\section{Acknowledgements}

This work is supported by National Social Science Fund (Grant No. 14BGL062), the National Science \& Technology Pillar Program during the 12th Five-Year Plan Period (Grant No. 2014BAL07B05). 


\section{References}

[1] K. Zhu, and R. Q. Zhang, et al.: Management Science, 53 (2007) 3, 421-436.

[2] Y. W. W. Mand: Journal of Industrial Engineering and Engineering Management, (2004), 96-98.

[3] R., A. M. S. P.: the 78th EAAE Seminar and NJF Seminar330 (Copenhagen, 2001).

[4] Tapiero, C. S.: European Journal of Operational Research, 2 (2007) 182, 683-694.

[5] K, H. V. K., Hazard M.: European Review of Agricultural Economics, (2002), 271-279.

[6] Chao, G. H. and S. M. R. Iravani, et al.: Management Science, 55 (2009) 7, 1122-1138.

[7] E. Katok and E. Siemsen: Management Science, 57 (2011) 6, 1042-1054.

[8] Yeung, A. C. L.: Journal of Operations Management, 4 (2008) 26, 490-502.

[9] T., J.: Mathematic Bioscience, (1978), 145-156.

[10]D. Estampe, and S. Lamouri, et al.: A Framework for Analysing Supply Chain Performance Evaluation Models, (2011).

[11] J. Sun, and M. Matsui, et al.: Supplier Risk Management: An Economic Model of P-chart Considered Due-date and Quality Risks, (2012). 